\title{
Improving Education Opportunity, Health, and Quality of Life of Orphans and Vulnerable Children: The Sustainable Action against HIV and AIDS in Communities (SAHACOM)
}

Siyan Yi

Touro University California, siyan@doctor.com

Pheak Chhoun

Samantha Brant

Touro University California

Kelley Kita

Touro University California

Sovannary Tuot

Follow this and additional works at: https://touroscholar.touro.edu/cehs_pubs

Part of the Immune System Diseases Commons, International Public Health Commons, and the Maternal and Child Health Commons

\section{Recommended Citation}

Yi, S., Chhoun, P., Brant, S., Kita, K., \& Tuot, S. (2015). Improving education opportunity, health, and quality of life of orphans and vulnerable children: The Sustainable Action against HIV and AIDS in Communities (SAHACOM). International Journal of Tropical Disease and Health, 5(1), 37-47.

This Article is brought to you for free and open access by Touro Scholar. It has been accepted for inclusion in by an authorized administrator of Touro Scholar. For more information, please contact touro.scholar@touro.edu. 


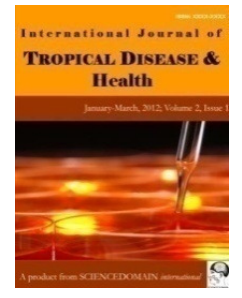

International Journal of TROPICAL DISEASE \& Health

5(1): 37-47, 2015, Article no.IJTDH.2015.005

ISSN: 2278-1005

SCIENCEDOMAIN international

www.sciencedomain.org

\title{
Improving Education Opportunity, Health, and Quality of Life of Orphans and Vulnerable Children: The Sustainable Action against HIV and AIDS in Communities (SAHACOM)
}

\author{
Siyan $\mathrm{Yi}^{1,2^{*}}$, Pheak Chhoun ${ }^{1}$, Samantha Brant ${ }^{2}$, Kelley Kita ${ }^{2}$ \\ and Sovannary Tuot ${ }^{1}$ \\ ${ }^{1}$ Research Department, KHANA, Cambodia. \\ ${ }^{2}$ Public Health Program, College of Education and Health Sciences, Touro University, California, \\ United States.
}

Authors' contributions

This work was carried out in collaboration between all authors. Authors SY managed the literature review, designed the study, developed the research protocol, analyzed the data, and wrote the first draft the manuscript. Authors PC, SB, KK and ST were responsible for data collection and supported study design, protocol development, and analyses of the study findings. All authors read and approved the final manuscript.

Article Information

DOI: 10.9734/IJTDH/2015/13535 Editor(s):

(1) Anthony R. Mawson, Public Health \& Director Institute of Epidemiology \& Health Services Research, Jackson State University, USA. Reviewers:

(1) J. Anitha Menon, Department of Psychology, University of Zambia, Zambia.

(2) Anonymous, USA

(3) Okpokoro Evaezi, Research Department, Institute of Human Virology, Nigeria. Complete Peer review History: http://www.sciencedomain.org/review-history.php?iid=711\&id=19\&aid=6760

Original Research Article

Received 21 $1^{\text {st }}$ August 2014

Accepted $5^{\text {th }}$ October 2014

Published $5^{\text {th }}$ November 2014

\section{ABSTRACT}

Aims: This study evaluated the impact of the Sustainable Action against HIV and AIDS in Communities (SAHACOM) project in improving education opportunity, health, and quality of life of orphans and vulnerable children (OVC) in Cambodia.

Study Design: Operational intervention study.

Place and Duration of the Study: Five provinces in Cambodia, from 2010 to 2014.

Methodology: A two-stage cluster sampling method was used to select $756 \mathrm{OVC}$ at midterm and 
785 OVC at end line for face-to-face interviews. Outcome indicators from end line (2014) were compared to those obtained at midterm (2012). Where possible, the data were also compared with data obtained from baseline documentation (2010).

Results: The percentage of OVC receiving external support for child care surged from $30.0 \%$ at baseline to $84.0 \%$ at midterm but decreased to $76.8 \%$ at end line. Compared to children at midterm, children at end line were significantly more likely to report having attended school regularly in the past 12 months, less likely to suspend study in order to work to help feed the family in the past 12 months, less likely to respond that food supports and other basic needs such as clothes and other household materials were the most important needs for their family today, more likely to perceive that supports for child education were the most important for their family today, less likely to report that their family reduced times for daily meals due to the shortage of food in the past 12 months, and more likely to rate their general health and overall quality of life as fair, good, or very good.

Conclusion: This study indicates significant impact of the SAHACOM on education, health, and quality of life of OVC in Cambodia. This community-based model should be adapted for future interventions, taking into account the available resources.

Keywords: Community-based intervention; education opportunity; overall health status; HIV/AIDS; orphans and vulnerable children; quality of life; sustainability; Cambodia.

\section{INTRODUCTION}

HIV/AIDS continues to be a major global public health issue, having claimed more than 39 million lives so far [1]. As a result, 17.8 million children under the age of 18 have been orphaned by AIDS, and this will rise to 25 million by 2015 [2]. Consequently, the term Orphans and Vulnerable Children (OVC) has surfaced. This term refers to both orphans and other children who are more exposed to risks than their peers. According to the World Bank, OVC can be grouped into several categories [3]. These categories include street children, children in the worst forms of child labor, children affected by armed conflict, children affected by HIV/AIDS, children living with disability, children in multiple OVC categories, and local OVC groups. Local OVC groups are children who are considered vulnerable in their own local context such as child substance abusers in South Africa and trafficked children in Benin [4].

HIV/AIDS introduce unprecedented challenge to OVC in communities, threatening their health, education, and quality of life [5]. The survival and development of a child is at risk if the family environment is undermined as a result of illness or death of the parents or guardians. If the mother or guardian of a child dies during his or her first year of life, this might have lifethreatening impact on the child's life. Resulting changes in OVC's well-being are cumulative, multifaceted, and interlinked [5]. The changes may include increased risks of school drop-out and poor school performance [6-8], impaired food security [9-11], diminished psychological wellbeing [12-14], elevated risks of HIV and sexually transmitted infections [15-17], and reduced access to health care and other services [18-20]. The economic and social effects on OVC may imply reduced access to education and health care [21,22]. Children whose mothers are infected with HIV are significantly more likely to be stunted, wasted, or underweight compared to their counterparts of similar demographic and socio-economic background whose mothers are not infected [23]. Findings from a systematic review indicate the value of community-based interventions in improving OVC's wellbeing and suggest that a strategic research agenda is needed to inform resource allocation and program management decisions [5].

KHANA is the largest national non-governmental organization in Cambodia working through a strong network of community-based implementing partners to provide integrated HIV prevention, care, and support services at the community level. Since 2009, KHANA has implemented the Sustainable Action against HIV and AIDS in Communities (SAHACOM) through 20 community-based implementing partners in the capital city and eight provinces providing care and support for OVC as part of the Integrated and Prevention Program. As a member of the OVC National Task Force and Working Groups for Child Welfare and Child Survival, KHANA and its implementing partners had been providing a comprehensive package of care which includes improvement of access to formal education, psychological support, social welfare support, health promotion, vocational trainings, and 
improvement of access to basic medical services including referral to antiretroviral therapy. It is vital that this package is strengthened to respond to the growing needs of OVC.

The SAHACOM utilizes a community-based approach to empower and create ownership among the communities being served. Through this model, community support volunteers, peer facilitators, and peer educators are utilized to provide support, services, and implement the activities of the project. It aims to reduce the impact of HIV/AIDS by improving health and quality of life of the most vulnerable populations including OVC. The implementing partners have also been provided technical supports on capacity building as well as policy and strategy work. By September 2013, a total of 15,681 OVC had been reached through KHANA's communitybased care activities, and 1,791 HIV-positive children had been supported to access clinical services [24]. The logical framework for the SAHACOM is shown in the Fig. 1.

Studies in different settings have shown the effectiveness of community-based intervention models in improving access to adequate food, school enrolment, and access to healthcare services $[5,25]$. However, preliminary analyses of a community-based intervention in Zambia and Rwanda indicated that the intervention was not effective in getting children into school at an earlier age, but was successful at keeping children in school longer despite secular trends in the opposite direction [26-28]. To address the gaps in the literature, further investigations and reports are needed [5]. We therefore conducted this study to measure the effectiveness of the SAHACOM by examining the extent to which the intended objectives of the project set at the baseline have been achieved. The specific objectives of the study were to assess the changes of key outcome indicators including education opportunity, food security, and general health and quality of life among OVC from baseline to end line and to document lessons learned and challenges experienced during five years of the project life. Findings from this study would be necessary for review and revision of the project implementation for further improvement of the target outcomes in Cambodia as well as in other resource-poor settings.

We hypothesized that the SAHACOM is effective in improving education opportunity, food security, and general health and quality of life of OVC in communities in Cambodia. In this study, education opportunity was indicated by proportion of OVC who were currently in school, who were able to attend school regularly, and who did not have to suspend study in order to work for feeding the family. Food security was indicated by proportion of OVC whose family had sufficient food to eat and did not need to reduce times of daily meals because of the shortage of food. Regarding health and quality of life, the overall conditions were indicated by the proportion of OVC who reported their overall health and quality of life as fair, good, or very good.

\section{MATERIALS AND METHODS}

Shortly after the project was started in 2009 , the SAHACOM baseline documentation was conducted using desk reviews, field visits, and consultative meetings with various program staff, and a number of core indicators and practical recommendations were documented. A mid-term and end-line survey was conducted in 2012 and 2014, respectively.

\subsection{Study Sites and Sampling}

Participants were recruited from five provinces including Battambang, Pailin, Pursat, Siem Reap, and Takeo. The $z$ test for a two-sample comparison of proportions was employed to detect a change of $10-15 \%$ of key indicators such as self-rated general health and quality of life. The power of $80 \%$ with $95 \%$ confidence interval was set for the calculation. Design effect of 2 was used to compensate the cluster effect. The selection of these indicators was based on the SAHACOM baseline documentation report [29] and findings from the SAHACOM midterm review [30]. As a result, the total minimum sample size required for the study was approximately 690 samples. Adjusted for incomplete responses and missing data of $10 \%$, the final sample size was approximately 760 .

Five out of nine provinces where interventions targeting OVC have been implemented were selected to represent the overall SAHACOM. We excluded other provinces because the target population size for the interventions was too small at the time when the baseline documentation and mid-term review was conducted. Moreover, the total number of the population in the selected five provinces represented more than $70 \%$ of the total coverage of the project activities. 


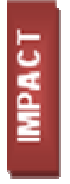

Improved health \& quality of life of people in Cambodia by reducing the impact of HIV and AIDS, especially amongst the most vulnerable population groups

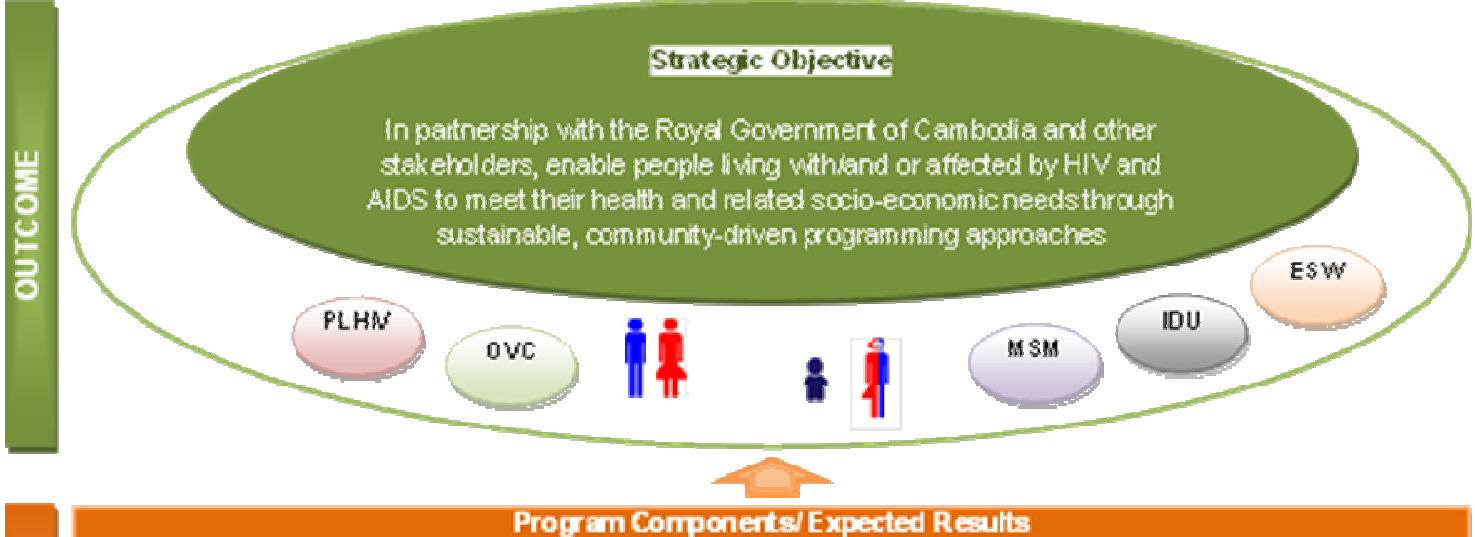

ER1. Improved coverage, quality \& sustainability of con prehensive and irtegrat services for PLHV (induding

KPS) and OVC, which have suocesstully linked communities with public health and non-health services

ER2. Improved uptake of imovative and targeted HV prevention interventions and services to KPs, with a particular focus on under-served and negeded groups

ER3. Strengthened capacity and leadership of NGO siCBOs and communities (especially thcse representing KPs \& PLHIVleads to their meaninaful particination in deliverina qualitv and sustainable communitv-based HIV
\begin{tabular}{|ll} 
Irtermediate Restits \\
IR1.1 Full coverage achieved andmaintained in projed sites of high quality comprehensive care, treatment and
\end{tabular} support services for PLHIV (induding KPs) and OVC
IR1.2 Improved integration of HIVrelated services for health, social welfare and in pact mitigation with existing hane \& comm unity-based care services

IR1.3 Increased capacity and sustainability of selfthelp groups

IR2.1 Increased access to services, HIV knowedge \& related behavior change anong KPS

IR2.2 Supportive enwronment established for HV prevention programming with KPs at national and sub-naticnal levels

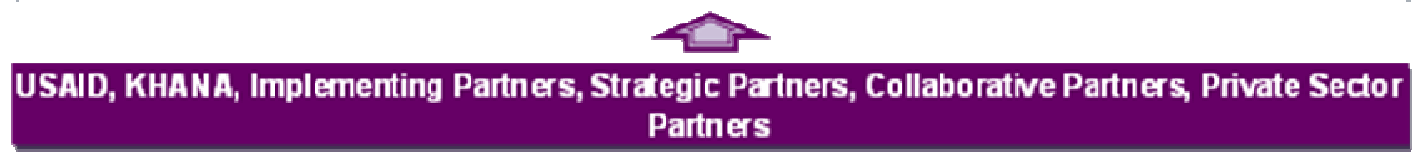

Fig. 1. Logical framework for the SAHACOM project (adapted from the SAHACOM End-ofproject evaluation reported by an external evaluation team of the USAID/Cambodia)

We used a two-stage cluster sampling method to select the study samples. The sample size was proportionally allocated to the size of OVC under the SAHACOM in each province. Number of health centers in each selected province to be included in the study was decided based on the number of OVC registered in each health center, where basic HIV care and treatment services had been provided to OVC. To be included, a health center should cover at least 20 OVC. We assessed several other factors when deciding whether to include a health center in the study 
such as number of people living with HIV, convenience for data collection, and duration of the project implementation in the health center coverage. We used the probability proportionalto-size sampling to decide the required number of OVC in each health center. Participants were then randomly selected from a name list of OVC under the coverage of the selected health center obtained from a community-based organization responsible for the implementation of the SAHACOM in the coverage area.

\subsection{Data Collection Training Procedures}

A two-day training was provided to all interviewers and field supervisors. The main objective of the training was to ensure that all interviewers and field supervisors understood the procedures and followed the standardized guideline in the same manner and guaranteed the quality of the data. The training covered necessary skills including interview techniques, confidentiality, and practices of the questionnaire administration. We also reviewed the study protocol during the training sessions in order for the team members to be thoroughly familiarized with it. Quality control skills such as rechecking and reviewing the questionnaires after administration as well as resolving issues that might arise during the fieldwork were included in the training. Regular review sessions with interviewers were conducted during the survey period to review progress and communicate any issues occurring during the data collection.

\subsection{Questionnaire Development}

Standardized tools were adapted from previous studies to measure key outcome indicators related to the project objectives. We initially developed the questionnaire in English and then translated it into Khmer, the national language of Cambodia. It was then back-translated into English by another translator to ensure that the "content and spirit" of every original item were maintained. Clear instructions and explanations were addressed to avoid any confusion during the interviews.

A pilot study was conducted prior to the main data collection to ensure that the wording and contents were culturally suitable, acceptable, and clearly understandable for the study participants. The pilot study was conducted with 10 randomly selected samples to assess the contents, format, length, language, and appropriateness of the questionnaire. Necessary modifications were made based upon feedbacks from the pilot study and comments from researchers and practitioners working on this area in Cambodia.

\subsection{Variables and Measurements}

Data were collected through an interview with the OVC themselves if he/she was 11 years of age or older and with his/her caregiver if he/she was younger than 11 years. Socio-economic characteristics, community support, and health and nutrition status were measured using existing items adapted from our previous studies in the same populations [31] and the most recent Cambodia Demographic and Health Survey [32].

Socio-economic characteristics included age, gender, maternal and paternal occupation, main caregiver or guardian, schooling status, years of schooling completed, whether they had enough stationary for their study in the past six months, whether they had ever suspended school to work for feeding their family in the past six months, and whether they had received any external support for schooling as well as sources of the support received in the past six months. For those who were not currently in school, they were also asked about the reasons for not being currently in school.

Regarding community support, the children were asked whether they or their family had received any external support after their parents passed away or got sick. Yes/no questions were asked to collect information about receipt of different types of support in the past 12 months. The respondents were also asked about sources of the support they had received, types of support they thought the most useful for them or their family, and whether they thought their family life situation would be more difficult if there was no food support.

For health and nutrition, children were asked about their HIV status, their overall health status and quality of life, whether they had been very sick making them unable to work or go to school, and duration of the sickness in the past six months. Self-rated general health status was measured using the following question, "In general, how would you rate your overall health?" with five response options including very good, good, neither good nor poor, poor, and very poor. Similarly, self-rated quality of life was measured using the following question, "In general, how would you rate your overall quality of life?" with five response options including very good, good, 
neither good nor poor, poor, and very poor. Regarding food security, we collected information on number of meal times the children had on an average day, whether the children had sufficient food to eat and the reason for not having sufficient food in the past six months, whether their family had reduced the number of meal times because of not having sufficient food, and if they thought that their family had less amount of food than that of other families in the community.

\subsection{Ethical Consideration}

Participation in this study was voluntary. Because all OVC were under the age of 18, an informed consent was obtained from their caregivers or guardians, such as relatives or NGO staff who took care of them, after a detailed description of the study objectives and procedures had been provided. Caregivers, guardians, or OVC themselves could refuse or discontinue the participation at any time for any reason without any consequences. Confidentiality of the information was ensured by removing all personal identifiers from the survey questionnaires. Data were computer coded and kept under the responsibility of the research team leaders of KHANA. The study protocol was approved by the National Ethics Committee for Health Research, Ministry of Health, Cambodia.

\subsection{Data Entry and Analyses}

Data were coded and entered into a computerized database using Epi Data version 3 (Odense, Denmark). Double data entry was performed to minimize entry errors. Descriptive statistical tests were used to compute means and standard deviations (SD) for numerical variables and frequencies for nominal and ordinal variables. To test the significance of the changes, we used McNemar's test for categorical variables and paired Student's $t$-test for continuous variables to compare key outcome indicators at midterm to key outcome indicators at end line. Two-sided $p$-values of less than 0.05 were regarded as statistically significant. SPSS version 20.0 (IBM Corporation, New York, USA) was used for all data analyses.

\section{RESULTS}

\subsection{Socio-economic Characteristics of OVC at End Line}

In total, 756 OVC were included in midterm survey and 785 OVC in end-line survey. At end line, approximately half $(48.6 \%)$ of the children were boys with the mean age of 12.6 years $(\mathrm{SD}=$ $2.7)$. The majority of the children $(77.0 \%)$ lived in their own family house, while the remaining lived in their relative's house (19.8\%), other people's house or rented house $(2.4 \%)$, and other places such as an orphanage, pagoda, or public shelter $(0.8 \%)$. The average number of siblings of the respondents was $3.1 \quad(S D=1.7)$, and their caregiver included parents (65.5\%), grandparents $(23.5)$, relatives $(6.5 \%)$, siblings $(2.4 \%)$, or other $(2.1 \%)$. Less than a quarter $(20.4 \%)$ of the respondents was living with HIV.

Regarding their education, the majority of the children were currently in school (93.2\%). Among the students, only $48.8 \%$ reported having enough materials for study, and $9.0 \%$ reported having to suspend school in order to work to help feed the family in the past 12 months. The majority of the children $(81.2 \%)$ reported receiving some form of educational support in the past six months; and of them, $87.2 \%$ had received such supports from KHANA and its implementing partners. When asked about other living situations, 92.5\% reported having enough food to eat in the past 12 months, and only $5.1 \%$ reported that their family had reduced daily meal times due to the lack of food in the past 12 months. However, only $64.8 \%$ of them had three meals per day in the past 12 months.

The children or their family had received different types of social support since their parents died or got seriously sick including support for child education (81.2\%), financial support for starting up family business $(79.8 \%)$, clothes or other materials for daily living (70.7\%), food support $(63.7 \%)$, and psychological support such as home visits and counseling (58.7\%) from NGO staff and other people. When asked to raise the most important needs for their current family daily living, $80.9 \%$ of the children perceived school materials as the most important, followed by basic materials for daily living such as clothes and other household materials (61.2\%), financial support for starting up family business (60.3\%), food support (48.3\%), and psychological support such as home visits or counseling (12.6\%). 
Regarding their overall health conditions, $34.1 \%$ of OVC reported that they had been seriously sick preventing them from their daily work or study in the past three months. When asked to rate their health condition, $92.2 \%$ of the respondents reported that their overall health condition was fair, good, or very good, and $87.7 \%$ of them rated their overall quality of life as fair, good, or very good.

As shown in Table 1, no significant difference was found in the comparisons of socio-economic characteristics of the children at midterm and end line.

\subsection{Comparisons of Types of Supports}

Table 2 shows the comparisons of types of support received in the past 12 months among OVC at midterm and end line. Compared to children at midterm, children at end line were significantly less likely to receive several types of support from any sources such as food $(R R=1.2$, $95 \% \mathrm{Cl}=1.1-1.2)$, clothes and other household materials for daily living $(\mathrm{RR}=1.6,95 \% \mathrm{Cl}=1.5$ 1.8), financial support for starting up family business (RR=1.2, 95\% Cl= 1.1-1.2), and psychological support such as home visits or counseling ( $\mathrm{RR}=1.9,95 \% \mathrm{Cl}=1.7-2.0)$. They also were significantly less likely to receive support for child education from KHANA and its implementing partners in the past six months $(\mathrm{OR}=1.2,95 \% \mathrm{Cl}=1 \cdot 1-1.2)$. However, it is worthnoting that only $30.0 \%$ of OVC at baseline received overall external support for childcare compared to $84.0 \%$ and $76.8 \%$ at midterm and end line, respectively.

\subsection{Comparisons of Types of Support Perceived to be the Most Important}

Comparisons of types of support perceived to be the most important for today family daily living among OVC at midterm and end line are shown in Table 3. Children at end line were significantly less likely to respond that food support $(R R=1.6$, $95 \% \mathrm{Cl}=1.5-1.7)$ and other basic needs such as clothes and other household materials $(R R=1.2$, $95 \% \mathrm{Cl}=1.1-1.3)$ were the most important needs for their family today compared to children at midterm. However, children at end line were significantly more likely to perceive that support for child education were the most important need for their family life today $(\mathrm{RR}=1.8,95 \% \mathrm{Cl}=1.6$ 1.9) compared to children at midterm.

\subsection{Comparisons of Education, Nutrition, and Health Conditions}

Table 4 shows the comparisons of education opportunity and self-rated general health and quality of life of OVC at midterm and end line. Compared to children at midterm, children at end line were significantly more likely to report that they had attended school regularly $(R R=1.2$, $95 \% \mathrm{Cl}=1.1-1.3$ ) and less likely to suspend their study in order to work to help feed the family $(\mathrm{RR}=1.6,95 \% \mathrm{Cl}=1.2-2.1)$ in the past 12 months. The proportion of children who reported that their family had sufficient food to eat in the past 12 months was not significantly different. However, children at end line were significantly less likely to report that their family had reduced times for daily meals due to the shortage of food in the past 12 months $(\mathrm{RR}=5.1,95 \% \mathrm{Cl}=3.7$ 7.0). Regarding their self-rated health, children at end line were significantly more likely to rate their overall health as fair, good, or very good ( $R R=$ $1.2,95 \% \mathrm{Cl}=1.2-1.3)$ and overall quality of life as fair, good, or very good $(\mathrm{RR}=1.2,95 \% \mathrm{Cl}=$ 1.2-1.3) compared to children at midterm.

\section{DISCUSSION}

This study describes the impact of communitybased activities of the SAHACOM in improving education opportunity, food security, overall health, and quality of life of OVC in rural Cambodia. The proportion of OVC households that received basic external support in caring for the children increased significantly from baseline and midterm. However, there was a decrease in the basic support from midterm $(84.0 \%)$ to end line $(76.8 \%)$, but it remained much higher than the life-project target that was set at $50 \%$. This result shows the reality of the program implementation as it reduced some activities and supports to OVC in the last period of the project from fiscal year 2012 due to the limitation of the budget. Decline in supports received in the past 12 months from midterm to end line was shown in clothes and other materials for basic daily life, psychological supports, and financial supports to start up family business. The decrease and increase in areas of support are consistent with respondents' answers to what is most important areas of supports needed, demonstrating that needs assessment for this population is continuous and on-going. 
Table 1. Comparisons of socio-economic characteristics of OVC at midterm and end line

\begin{tabular}{|c|c|c|c|}
\hline Variables & Midterm & End line & $\operatorname{RR}(95 \% \mathrm{CI})$ \\
\hline Mean age of participants $( \pm S D)$ & $12.8 \pm 2.6$ & $12.6 \pm 2.7$ & 0.82 \\
\hline Male gender & $335(44.4)$ & $382(48.6)$ & $1.1(0.9-1.2)$ \\
\hline Living with HIV & $129(17.1)$ & $160(20.4)$ & $1.2(0.9-1.5)$ \\
\hline \multicolumn{4}{|l|}{ Main care giver/guardian } \\
\hline Parents & $485(64.2)$ & $515(65.5)$ & $1.0(0.9-1.1)$ \\
\hline Grandparents & $168(22.2)$ & $185(23.5)$ & $1.1(0.9-1.3)$ \\
\hline Siblings & $22(3.0)$ & $19(2.4)$ & $1.2(0.7-2.2)$ \\
\hline Other (relatives, step-parent, orphanage) & $72(9.5)$ & $67(8.5)$ & $1.1(0.8-1.5)$ \\
\hline Currently in school & $696(92.9)$ & $725(93.2)$ & $1.0(0.9-1.1)$ \\
\hline Having enough study materials & $416(59.1)$ & $355(48.8)$ & $1.2(1.0-1.3)$ \\
\hline
\end{tabular}

Table 2. Comparisons of types of support received by OVC at midterm and end line

\begin{tabular}{lccc}
\hline Variables & Midterm & End line & RR (95\% CI) \\
\hline Social support received in the past 12 months & & & \\
Food supports (rice, noodles, canned fish) & $614(81.2)$ & $597(76.1)$ & $1.2(1.1-1.2)$ \\
Clothes and other household materials & $668(88.4)$ & $428(70.7)$ & $1.6(1.5-1.8)$ \\
Financial supports for staring up family business & $637(84.3)$ & $483(79.8)$ & $1.2(1.1-1.2)$ \\
Psychological supports (home visits, counseling) & $639(84.5)$ & $353(58.7)$ & $1.9(1.7-2.0)$ \\
Support for child education from any sources & $616(81.5)$ & $638(81.2)$ & $1.0(0.9-1.1)$ \\
Support for child education from KHANA/IPs & $591(96.0)$ & $555(87.2)$ & $1.2(1.1-1.2)$ \\
\hline \multicolumn{4}{c}{ Abbreviations: Cl, confidence interval; IP, implementing partner; RR, risk ratio; OVC, orphans and vulnerable } \\
\multicolumn{2}{c}{ children }
\end{tabular}

Table 3. Comparisons of types of support perceived to be the most important among OVC at midterm and end line

\begin{tabular}{lrrr}
\hline Variables & Midterm & End line & RR (95\% Cl) \\
\hline Perceived the most important needs for family daily living & & \\
Food supports (rice, noodles, canned fish) & $583(77.1)$ & $380(48.3)$ & $1.6(1.5-1.7)$ \\
Clothes and other household materials & $540(71.4)$ & $481(61.2)$ & $1.2(1.1-1.3)$ \\
Financial supports for starting up family business & $432(57.2)$ & $474(60.3)$ & $1.1(0.9-1.2)$ \\
Psychological supports (home visits, counseling) & $81(10.7)$ & $99(12.6)$ & $1.2(0.9-1.6)$ \\
Support for child education & $345(45.7)$ & $636(80.9)$ & $1.8(1.6-1.9)$ \\
\hline
\end{tabular}

Abbreviations: $\mathrm{Cl}$, confidence interval; $R R$, risk ratio; OVC, orphans and vulnerable children

Table 4. Comparisons of education, food security, and health status among OVC at midterm and end line

\begin{tabular}{lccc}
\hline Variables & Midterm & End line & RR (95\% Cl) \\
\hline Attended school regularly in the past 12 months & $645(85.3)$ & $703(89.6)$ & $1.2(1.1-1.3)$ \\
Suspended study to work in the past 12 months & $108(14.3)$ & $71(9.0)$ & $1.6(1.2-2.1)$ \\
Had sufficient food to eat in the past 12 months & $697(92.6)$ & $725(92.5)$ & $1.0(0.9-1.1)$ \\
Reduced times of daily meals in the past 12 months & $196(25.9)$ & $40(5.1)$ & $5.1(3.7-7.0)$ \\
Having been seriously sick in the past 6 months & $222(28.4)$ & $265(33.8)$ & $1.1(1.0-1.3)$ \\
Self-rated overall health as fair, good, or very good & $574(86.1)$ & $724(92.2)$ & $1.2(1.2-1.3)$ \\
Self-rated overall quality of life as fair, good, very good & $549(82.3)$ & $694(87.7)$ & $1.2(1.2-1.3)$ \\
\hline \multicolumn{2}{c}{ Abbreviations: Cl, confidence interval; RR, risk ratio; OVC, orphans and vulnerable children }
\end{tabular}

Despite the withdrawal of food support from the World Food Programme (WFP) in December 2012, food support was no longer reported as the most urgent need in OVC's daily life among OVC at end line compared to that at midterm. Instead, support for child education was reported as the most important need for family daily living. The majority of the respondents reported that their 
family had sufficient food to eat in the past 12 months. However, future surveys might need to more carefully define what "sufficient" food is due to the fact that only $64.8 \%$ of respondents reported having three meals a day in the past 12 months, illustrating a slight decrease compared to midterm where $71.3 \%$ of the respondents reported having three meals a day. A possible explanation for the slight decrease rather than a dramatic decrease in OVC who had three meals a day could be due to KHANA's continuous efforts to provide food support to the most vulnerable OVC post closure of the WFP's program. These statistics are important to identify that, although almost all respondents said their family had enough food to eat in the past 12 months, more support may be necessary. Additional support may lead to better health for OVC and increase in school attendance, since $9.0 \%$ of the respondents reported having to stop going to school in order to work to help feed their family.

A noteworthy accomplishment is the increase of regular school attendance reported by OVC from $85.3 \%$ at midterm to $89.6 \%$ at end line. This can be attributed to an increase and continuous supports for child education. Of total, $68.1 \%$ of the children received educational support in the past six months, from this $87.2 \%$ received this support from KHANA and its implementing partners. Moreover, school material supports in the past 12 months increased from $76.1 \%$ at midterm to $81.2 \%$ at end line. Child education seems to be more valued as illustrated by the increase in school attendance and the substantial increase shown when respondents were asked about the most important needs for daily family living, and support for child education were reported at $45.7 \%$ at midterm and $80.9 \%$ at end line.

The increased value of education could be due to the fact that OVC and their family were receiving social supports, allowing for opportunities that were not previously available. In a complementary in-depth interview, a provincial AIDS program officer reported that, due to the services provided, there had been a decline in people migrating in order to find money or food elsewhere. Less migration may allow families to think more about the future, especially in terms of child education. In addition, through the SAHACOM, work had been done to reduce discrimination and stigma faced by OVC and increase self-esteem in OVC, which could result in OVC enjoying school more. In turn, their quality of life had also been improved.
Several limitations of this study should be noted. First, as with any self-reported measures, there are inherent biases and potential for both underreporting and over-reporting in the variables. Second, for this end-of-project evaluation, baseline survey was not conducted, and comparisons of key indicators were made using data from desk-review documentation in which only a few indicators for OVC were available. This condition made the measurements of changes of the key indicators from baseline to end line difficult. Third, findings from this study might be limited by unknown reliability and validity of the questionnaire. However, the questionnaire was developed using items adapted from previous studies in Cambodia and carefully reviewed by local and international experts and pretested before the final version was constructed. The final limitation concerns possible recall biases as participants were asked to recall events that had taken place over the past several months.

\section{CONCLUSION}

Findings from this study indicate significant impact of the SAHACOM on education, food security, health, and quality of life of OVC in Cambodia. The increase in perceived importance of child education and school attendance rate indicates the importance of sustaining this support in the future for OVC population. It is also important to consider factors that could potentially keep children from regular attendance including having to work to help feed the family, migration in hopes of financial opportunities elsewhere, and high quality access to health care services to prevent kids from being too sick to go to school.

A challenge that was continuously demonstrated throughout the SAHACOM project was the high turnover of staff of the implementing partners. High turnover rates resulted in additional energy in recruitment and hindered capacity building and integration of services. In order to decrease the rate of turnover in the future, it is necessary to increase incentives for their work. The implementing partners are an important component in serving the target population as they work to organize community awarenessraising and small group sessions to increase their understanding, referrals for healthcare services, and address the unmet needs of the children. 


\section{CONSENT}

An informed consent was obtained from caregivers or guardians of each participant after a detailed description of all study objectives and procedures had been provided.

\section{ETHICAL APPROVAL}

All authors hereby declare that all experiments have been examined and approved by the appropriate ethics committee and have therefore been performed in accordance with the ethical standards laid down in the 1964 Declaration of Helsinki.

\section{COMPETING INTERESTS}

Authors have declared that no competing interests exist.

\section{REFERENCES}

1. World Health Organization (WHO). Factsheet on HIV/AIDS. Geneva, Switzerland: WHO; 2014.

2. AIDS Orphans. HIV and AIDS. London, the United Kingdom: AIDS Orphans; 2013.

3. Kielland A. The World Banks' OVC Thematic Group. Orphans and vulnerable Children (OVC). Washington DC, USA: World Bank; 2004.

4. World Bank. A Toolkit on how to support Orphans and Other Vulnerable Children in Sub-Saharan Africa. Washington DC, USA: World Bank; 2014.

5. Schenk KD. Community interventions providing care and support to orphans and vulnerable children: a review of evaluation evidence. AIDS Care. 2009;21:918-42.

6. Ainsworth $\mathrm{M}$, Beegle K, Koda $\mathrm{G}$. The impact of adult mortality and parental deaths on primary schooling in northwestern Tanzania. J Dev Stud. 2005;41:412-39.

7. Case A, Ardington C. The impact of parental death on school outcomes: Longitudinal evidence from South Africa. Demography. 2006;43:401-20.

8. Tu X, Lv Y, Li X, Fang X, Zhao G, Lin X, et al. School performance and school behavior of children affected by AIDS in China. Vulnerable Child Youth Stud. 2009;4:199-209.

9. Mishra V, Arnold F, Otieno F, Cross A, Hong $R$. Education and nutritional status of orphans and children of HIV-infected parents in Kenya. AIDS Educ Prev. 2007;19:383-95.

10. Nyambedha EO, Wandibba S, AagaardHansen J. Policy implications of the inadequate support systems for orphans in western Kenya. Health Policy. 2001;58:8396.

11. Rivers J, Mason JB, Rose DD, Eisele TP, Gillespie S, Mahy M, et al. The impact of orphanhood on food security in the highHIV context of Blantyre, Malawi. Food Nutr Bull. 2010;31(3):264-71.

12. Lester $P$, Rotheram-Borus MJ, Lee SJ, Comulada S, Cantwell V, Wu N, et al. Rates and predictors of anxiety and depressive disorders in adolescents of parents with HIV. Vulnerable Child Youth Stud. 2006;1:81-101.

13. Marais L, Sharp C, Pappin M, Rani K, Skinner D, Lenka M, et al. Communitybased mental health support for orphans and vulnerable children in South Africa: A triangulation study. Vulnerable Child Youth Stud. 2014;9:151-58.

14. Nostlinger C, Bartoli G, Gordillo V, Roberfroid D, Colebunders R. Children and adolescents living with HIV positive parents: Emotional and behavioural problems. Vulnerable Child Youth Stud. 2006;1:29-43.

15. Gregson S, Nyamukapa CA, Garnett GP, Wambe M, Lewis JJC, Mason PR, et al. HIV infection and reproductive health in teenage women orphaned and made vulnerable by AIDS in Zimbabwe. AIDS Care. 2005;17:785-94.

16. Juma M, Askew I, Ferguson A. Situation analysis of the sexual and reproductive health and HIV risks and prevention needs of older orphaned and vulnerable children in Nyanza Province, Kenya. Nairobi: Population Council; 2007.

17. Thurman TR, Brown L, Richter L, Maharaj $P$, Magnani R. Sexual risk behavior among South African adolescents: Is orphan status a factor? AIDS Behav. 2006;10:62735.

18. Andrews G, Skinner D, Zuma K. Epidemiology of health and vulnerability among children orphaned and made vulnerable by HIV/AIDS in sub- Saharan Africa. AIDS Care. 2006;18:269-76.

19. Fawzi MC, Holman SR, Kiley R, Li M, Barry D, Bandara S, et al. Closing the implementation gap in services for children affected by HIV/AIDS: from assisting orphans and vulnerable children (OVC) to providing long-term opportunities for 
economic growth. J Health Care Poor Underserved. 2011;22:1401-12.

20. Miller CM, Gruskin S, Subramanian SV, Rajaraman D, Heymann SJ. Orphan care in Botswana's working households: Growing responsibilities in the absence of adequate support. Am J Public Health. 2006;96:1429-35.

21. Kürzinger ML, Pagnier J, Kahn JG, Hampshire R, Wakabi T, Dye TD. Education status among orphans and nonorphans in communities affected by AIDS in Tanzania and Burkina Faso. AIDS Care. 2008;20:726-32.

22. Oleke C, Blystad A, Fylkesnes K, Tumwine JK. Constraints to educational opportunities of orphans: a communitybased study from northern Uganda. AIDS Care. 2007;19:361-8.

23. Magadi MA. Cross-national analysis of the risk factors of child malnutrition among children made vulnerable by HIV/AIDS in sub-Saharan Africa: evidence from the DHS. Trop Med Int Health. 2011;16:570-8.

24. KHANA. Advancing technical experience for better service delivery: Annual report 2013. Phnom Penh, Cambodia. KHANA; 2014.

25. World Vision. World Vision core HIV and AIDS models operations research summary. World Vision Newsletter. Lusaka, Zambia and Johannesburg, South Africa; 2007.

26. Chatterji $M$, Dougherty, $L$ Ventimiglia $T$, Mulenga $\mathrm{Y}$, Jones A, Mukaneza $\mathrm{A}$, et al. The well-being of children affected by HIV/AIDS in Lusaka, Zambia, and
Gitarama Province, Rwanda: findings from a study. Washington, DC: Community REACH Program, Pact; 2005.

27. Chatterji M, Murray N, Buek K, Hutchinson $\mathrm{P}$, Mulenga $\mathrm{Y}$, Ventimiglia $\mathrm{T}$. Evaluating the impact of community-based interventions on schooling outcomes among orphans and vulnerable children in Lusaka, Zambia. United African Population (UAPS) Conference, Arusha, Tanzania; 2009.

28. Dougherty L, Forsythe V, Winfrey W, Buek $\mathrm{K}$, Chatterji M. A costing analysis of community based programs for children affected by HIV/AIDS: Results from Zambia and Rwanda. Washington, DC: Community REACH Program, Pact; 2005.

29. Heng S, Tuot S, Ung $M$. Baseline documentation: The sustainable action against HIV and AIDS in communities (SAHACOM). Phnom Penh, Cambodia: KHANA; 2011.

30. Heng $S$, Tuot $S$. Mid-term review of the sustainable action against HIV and AIDS in communities (SAHACOM). Phnom Penh, Cambodia: KHANA; 2013.

31. Heng S, Chhea C, Tuot S. Midterm review of the integrated care and prevention project regarding PLHIV and OVC. Phnom Penh, Cambodia: KHANA; 2010.

32. National Institute of Public Health, National Institute of Statistics and ORC Macro. Cambodia Demographic and Health Survey 2010. Phnom Penh, Cambodia and Calverton, Maryland, USA: National Institute of Public Health, National Institute of Statistics, and ORC Macro; 2010.

(c) 2015 Yi et al.; This is an Open Access article distributed under the terms of the Creative Commons Attribution License (http://creativecommons.org/licenses/by/4.0), which permits unrestricted use, distribution, and reproduction in any medium, provided the original work is properly cited.

Peer-review history:

The peer review history for this paper can be accessed here: http://www.sciencedomain.org/review-history.php?iid=711\&id=19\&aid=6760 